\title{
SAMPLING AND RECONSTRUCTION IN SPARSE ATOMIC SPACES
}

\author{
Volker Pohl, Ezra Tampubolon, and Holger Boche \\ Lehrstuhl für Theoretische Informationstechnik \\ Technische Universität München, 80290 München, Germany \\ \{volker.pohl, ezra.tampubolon, boche\}@tum.de
}

\begin{abstract}
This paper provides a quantitative notion of the sparsity for infinite dimensional atomic spaces, which play an important role in many signal processing applications. This notion of sparsity is defined as the ratio of the number of redundant samples (not necessary to recover any signal in the atomic space) to the number of all available samples of a particular canonical sampling system. It is shown that the so defined sparsity can be expressed in terms of the support of the spectral density of the sequence which generates the atomic space.
\end{abstract}

Index Terms - Atomic spaces, redundancy, sampling, sparsity, stationary sequences

\section{INTRODUCTION}

In many practical signal processing applications signals are assumed to belong to so called shift-invariant spaces [1] $\mathcal{V}=\overline{\operatorname{span}}\left\{\phi_{n}(t)=\right.$ $\phi(t-n a): n \in \mathbb{Z}\}$. The sequence $\phi_{n}(t)=\phi(t-n a)=\left(\mathrm{R}_{a}^{n} \phi\right)(t)$ which spans $\mathcal{V}$ is generated by $\phi \in L^{2}(\mathbb{R})$ and by the translation operator $\mathrm{R}_{a}: \phi(t) \mapsto \phi(t-a)$ in $L^{2}(\mathbb{R})$. An obvious generalization of this signal model is obtained by replacing $L^{2}(\mathbb{R})$ with an arbitrary separable Hilbert space $\mathcal{H}$ and by replacing the translation operator $\mathrm{R}_{a}$ with an arbitrary unitary operator $\mathrm{U}$ in $\mathcal{H}$. Then the subspace $\mathcal{A}_{\phi} \subset \mathcal{H}$ of the form

$$
\mathcal{A}_{\phi}=\overline{\operatorname{span}}\left\{\phi_{n}=\mathrm{U}^{n} \phi: n \in \mathbb{Z}\right\}
$$

is called an atomic space [2]. In most applications of such a signal model, it is assumed that the sequence $\phi=\left\{\phi_{n}\right\}_{n \in \mathbb{Z}}$ forms a Riesz basis for $\mathcal{A}_{\phi}$. Then every $x \in \mathcal{A}_{\phi}$ has the form

$$
x=\sum_{n \in \mathbb{Z}} a_{n} \phi_{n}
$$

where $\boldsymbol{a}=\left\{a_{n}\right\}_{n \in \mathbb{Z}} \in \ell^{2}$ is a coefficient sequence uniquely determined by $x$. However, if $\phi$ is only a frame for $\mathcal{A}_{\phi}$ then the sequence $\boldsymbol{a}$ of the representation (2) of $x \in \mathcal{A}_{\phi}$ is no longer unique. This nonuniqueness of the coefficients introduces some redundancy in the representation of signals in $\mathcal{A}_{\phi}$, which may be used, e.g., to obtain an increased robustness against errors in the observation of $x \in \mathcal{A}_{\phi}$. Therefore, the assumption that $\left\{\phi_{n}\right\}_{n \in \mathbb{Z}}$ forms a frame (but not a Riesz basis) for $\mathcal{A}_{\phi}$ is often more appropriated.

Moreover, in the framework of generalized sampling, the sampling of a signal $x \in \mathcal{A}_{\phi}$ can be written as evaluations of inner products $\left\langle x, s_{n}\right\rangle$ of $x$ with a set $s=\left\{s_{n}=\mathrm{U}^{n} s\right\}_{n \in \mathbb{Z}}$ of sampling functions in $\mathcal{H}$, which is assumed to has the same structure as the sequence $\phi$ which spans the signal space $\mathcal{A}_{\phi}$. If $\mathcal{A}_{\phi}$ is assumed to be spanned by a Riesz basis sequence, then it is well known how

This work was supported by the German Research Foundation (DFG) under Grant BO 1734/22-1 and by the start-up fund of the TU München. any signal $x \in \mathcal{A}_{\phi}$ can be reconstructed from its samples $\left\langle x, s_{n}\right\rangle$, and which conditions the sequence $s$ has to satisfy, such that such a signal recovery is possible $[2,3]$.

In this paper, we assume that $\mathcal{A}_{\phi}$ is spanned by an over complete frame sequence and we derive conditions on $s$ such that signal recovery is possible. Moreover, since $\phi$ is overcomplete, the redundancy in the signal representation (2) can be used to reconstruct signals in $\mathcal{A}_{\phi}$ from only a subset $\left\{\left\langle x, s_{n}\right\rangle\right\}_{n \in I}$ of all available signal samples. Section 3 will quantify the amount of redundancy (or sparsity) $r\left(\mathcal{A}_{\phi}\right)$ contained in a signal space $\mathcal{A}_{\phi}$ of the form (1). Afterwards, it is shown that the sparsity $r\left(\mathcal{A}_{\phi}\right)$ of $\mathcal{A}_{\phi}$ indeed quantifies the necessary number of signal samples to reconstruct every $x \in \mathcal{A}_{\phi}$. Section 4 will illustrate a corresponding recovery scheme.

Another motivation for this work comes from recent results on the limitations of classical sampling schemes $[4,5]$. By considering more general sampling functionals and by exploiting the possible redundancy of the underlying signal space, it may be possible to overcome the convergence problems reported in these works, especially under additional quantization errors.

\section{SAMPLING IN ATOMIC SPACES}

Basic Notations We set $\mathcal{T}:=[0,2 \pi)$, and for $1 \leq p \leq \infty$ we write $L^{p}(\mathcal{T})$ for the usual Lebesgue space on $\mathcal{T}$. In particular, $L^{2}(\mathcal{T})$ stands for the Hilbert space of square integrable function on the interval $\mathcal{T}$ with the inner product

$$
\langle x, y\rangle=\frac{1}{2 \pi} \int_{0}^{2 \pi} x(\theta) \overline{y(\theta)} \mathrm{d} \theta
$$

If $\mathcal{Z}$ is a subset of $\mathcal{T}$, then $L^{2}(\mathcal{Z})$ will stand for the closed subspace of $L^{2}(\mathcal{T})$ of all those function which vanish outside of $\mathcal{Z}$ :

$$
L^{2}(\mathcal{Z})=\left\{f \in L^{2}(\mathcal{T}): f(\theta)=0 \text { a.e. } \theta \notin \mathcal{Z}\right\},
$$

and $\mathrm{P}_{\mathcal{Z}}$ stands for the orthogonal projection from $L^{2}(\mathcal{T})$ onto $L^{2}(\mathcal{Z})$. Every $f \in L^{2}(\mathcal{T})$ can be written as a Fourier series as

$$
f(\theta)=\sum_{n \in \mathbb{Z}} a_{n} \mathrm{e}^{\mathrm{i} n \theta} \quad \text { with } \quad a_{n}=\frac{1}{2 \pi} \int_{0}^{2 \pi} f(\theta) \mathrm{e}^{-\mathrm{i} n \theta} \mathrm{d} \theta
$$

and with the sequence $\boldsymbol{a}=\left\{a_{n}\right\}_{n \in \mathbb{Z}} \in \ell^{2}$ of Fourier coefficients. The (left) shift operator $\mathrm{S}: L^{2}(\mathcal{T}) \rightarrow L^{2}(\mathcal{T})$ is defined as

$$
\mathrm{S}: \sum_{n \in \mathbb{Z}} a_{n} \mathrm{e}^{\mathrm{i} n \theta} \mapsto \sum_{n \in \mathbb{Z}} a_{n+1} \mathrm{e}^{\mathrm{i} n \theta}
$$

or equivalently by $(\mathrm{S} f)(\theta)=f(\theta) \mathrm{e}^{-\mathrm{i} \theta}$. For any positive integer $Q$ the decimation operator $\mathrm{D}_{Q}: L^{2}(\mathcal{T}) \rightarrow L^{2}(\mathcal{T})$ is defined as

$$
\mathrm{D}_{Q}: \sum_{n \in \mathbb{Z}} a_{n} \mathrm{e}^{\mathrm{i} n \theta} \mapsto \sum_{n \in \mathbb{Z}} a_{Q n} \mathrm{e}^{\mathrm{i} n \theta}
$$

or equivalently as $\left(\mathrm{D}_{Q} f\right)(\theta)=\frac{1}{Q} \sum_{n=0}^{Q-1} f([\theta+n 2 \pi] / Q)$.

We will frequently need the notions of stationary sequences, frames, and Riesz bases in Hilbert spaces. Because of the limited space, we only refer to corresponding literature on these topics, e.g. $[6,7,8]$. 
Atomic Signal Spaces Let $\mathcal{H}$ be an arbitrary separable Hilbert space. We consider signals in atomic subspaces $\mathcal{A}$ of $\mathcal{H}$ [2]. These subspaces are characterized by a unitary operator $\mathrm{U}$ on $\mathcal{H}$ and by a generating function $\phi \in \mathcal{H}$. If we set $\phi_{n}:=\mathrm{U}^{n} \phi, n \in \mathbb{Z}$ then the atomic subspace $\mathcal{A}_{\phi}$ is defined as the closed linear span of the sequence $\phi:=\left\{\phi_{n}\right\}_{n \in \mathbb{Z}}$, i.e. by (1). It is always assumed that $\phi$ is a frame sequence, i.e. a frame for $\mathcal{A}_{\phi}$. Thus every signal $x \in \mathcal{A}_{\phi}$ has the form (2) with a coefficient sequence $\boldsymbol{a}=\left\{a_{n}\right\}_{n \in \mathbb{Z}} \in \ell^{2}$.

By its construction, $\phi=\left\{\phi_{n}\right\}_{n \in \mathbb{Z}}$ forms a stationary sequence in $\mathcal{H}$ [8], and since it is assumed to be a frame sequence, its autocorrelation function $r_{\phi}$ has the following spectral representation

$$
r_{\phi}(n-m):=\left\langle\phi_{n}, \phi_{m}\right\rangle=\frac{1}{2 \pi} \int_{0}^{2 \pi} \mathrm{e}^{\mathrm{i}(n-m) \theta} \Phi_{\phi}(\theta) \mathrm{d} \theta .
$$

Therein, $\Phi_{\phi} \in L^{1}(\mathcal{T})$ is called the spectral density of $\phi$. With every spectral density, we associate two disjoint subsets of $\mathcal{T}$ :

$$
\begin{aligned}
\mathcal{Z}_{\phi} & :=\left\{\theta \in \mathcal{T}: \Phi_{\phi}(\theta)=0 \text { a.e. }\right\} \\
\mathcal{M}_{\phi} & :=\left\{\theta \in \mathcal{T}: \Phi_{\phi}(\theta)>0 \text { a.e. }\right\} .
\end{aligned}
$$

The Lebesgue measure of both sets, will be denoted by $\lambda\left(\mathcal{Z}_{\phi}\right)$ and $\lambda\left(\mathcal{M}_{\phi}\right)$, respectively. The next lemma characterizes conditions on $\Phi_{\phi}$ such that $\phi$ forms a frame or Riesz basis for $\mathcal{A}_{\phi}$.

Lemma 1: Let $\phi=\left\{\phi_{n}\right\}_{n \in \mathbb{Z}}$ be a stationary sequence in $\mathcal{H}$ with spectral density $\Phi_{\phi}$. Then $\phi$ forms a frame for $\mathcal{A}_{\phi}=\overline{\operatorname{span}}\left\{\phi_{n}\right.$ : $n \in \mathbb{Z}\}$ with frame bounds $0<A \leq B<\infty$ if and only

$$
A \leq \Phi_{\phi}(\theta) \leq B \quad \text { a.e. } \theta \in \mathcal{M}_{\phi},
$$

and $\phi$ is a Riesz basis for $\mathcal{A}_{\phi}$ if and only if additional $\lambda\left(\mathcal{Z}_{\phi}\right)=0$.

Remark: A proof can be found in [8], and an extension to multidimensional stationary sequences in [3]. Moreover, for the particular case where $\phi$ is generated by the translation operator $\mathrm{U}=\mathrm{T}_{a}$ on $\mathcal{H}=L^{2}(\mathbb{R})$, the above result is very well known (see, e.g., [7, 9]).

With every frame sequence $\phi=\left\{\phi_{n}\right\}_{n \in \mathbb{Z}}$ one associates the synthesis operator $\mathrm{T}_{\phi}: \ell^{2} \rightarrow \mathcal{H}$ given by

$$
\mathrm{T}_{\phi}:\left\{a_{n}\right\}_{n \in \mathbb{Z}} \mapsto \sum_{n \in \mathbb{Z}} a_{n} \phi_{n} .
$$

We will need the following known characterization of the null space $\mathscr{N}\left(\mathrm{T}_{\phi}\right)$ of the synthesis operator $\mathrm{T}_{\phi}$.

Lemma 2: Let $\phi=\left\{\phi_{n}\right\}_{n \in \mathbb{Z}}$ be a stationary frame sequence in a Hilbert space $\mathcal{H}$ with spectral density $\Phi_{\phi}$ and with synthesis operator $\mathrm{T}_{\phi}$, then

$$
\mathscr{N}\left(\mathrm{T}_{\boldsymbol{\phi}}\right)=\left\{\boldsymbol{a} \in \ell^{2}: A(\theta)=\sum_{n \in \mathbb{Z}} a_{n} \mathrm{e}^{\mathrm{i} n \theta} \in L^{2}\left(\mathcal{Z}_{\boldsymbol{\phi}}\right)\right\} .
$$

We finally refer to [8] where several examples of stationary sequences on different Hilbert spaces are given and which are of some importance in applications. There it is also shown that it is often possible to determine explicitly the corresponding spectral density $\Phi_{\phi}$ in terms of the generator $\phi \in \mathcal{H}$.

Generalized Sampling The sampling of a signal $x \in \mathcal{H}$ is often described by an evaluation of inner products $c_{n}=\left\langle x, s_{n}\right\rangle$ with a set of sampling functions $\left\{s_{n}\right\}_{n \in \mathbb{Z}}$ in $\mathcal{H}$ [10], and where $\left\{c_{n}\right\}_{n \in \mathbb{Z}}$ are said to be the (generalized) samples of $x$. Here we consider Uinvariant sampling schemes [8] in which the sequence of sampling functions $\boldsymbol{s}=\left\{s_{n}\right\}_{n \in \mathbb{Z}}$ has the same structure as the sequence $\phi$ which spans our signal space $\mathcal{A}_{\phi}$, i.e. we assume that $s_{n}=\mathrm{U}^{n} s$, for all $n \in \mathbb{Z}$, with some generator $s \in \mathcal{H}$ and with the same unitary operator $U$ as in the definition (1) of $\mathcal{A}_{\phi}$. Similar as for $\phi$, the sequence $s$ is a stationary sequence with spectral density $\Phi_{s} \in L^{1}(\mathcal{T})$ and with two associated subsets $\mathcal{Z}_{s}$ and $\mathcal{M}_{s}$ of $\mathcal{T}$. Moreover, since both sequences $\phi$ and $s$ are generated by the same unitary operator $\mathrm{U}$, they are stationary correlated $[6,8]$ such that the corresponding cross-correlation function $r_{\phi, s}$ has the spectral representation

$$
r_{\phi, s}(n-m):=\left\langle\phi_{n}, s_{m}\right\rangle=\frac{1}{2 \pi} \int_{0}^{2 \pi} \mathrm{e}^{\mathrm{i}(n-m) \theta} \Phi_{\phi, s}(\theta) \mathrm{d} \theta
$$

with $\Phi_{\phi, s} \in L^{1}(\mathcal{T})$. Similar as above, we denote by $\mathcal{M}_{\phi, s}$ and $\mathcal{Z}_{\phi, s}$ the subsets of $\mathcal{T}$ where $\Phi_{\phi, s}$ is non-zero and equal to zero, respectively. It is not hard to see that $\mathcal{M}_{\phi, s}=\mathcal{M}_{\phi} \cap \mathcal{M}_{s}$ [11].

\section{THE SPARSITY OF ATOMIC SPACES}

Every $x \in \mathcal{A}_{\phi}$ can be written as $x=\mathrm{T}_{\phi} \boldsymbol{a}$ for some coefficient sequence $a \in \ell^{2}$. However, if $\phi$ is an overcomplete frame for $\mathcal{A}_{\phi}$ then there also exist other coefficient sequences $\widetilde{\boldsymbol{a}} \in \ell^{2}$ which yield the same $x \in \mathcal{A}_{\phi}$, i.e. for which $x=\mathrm{T}_{\phi} \boldsymbol{a}=\mathrm{T}_{\boldsymbol{\phi}} \widetilde{\boldsymbol{a}}$. One particular choice for such a sequence is $\widetilde{a}_{n}=\left\langle x, \widetilde{\phi}_{n}\right\rangle$ where $\left\{\widetilde{\phi}_{n}\right\}_{n \in \mathbb{Z}}$ is the so called canonical dual frame of $\phi$ (see, e.g., [7]). This sequence $\widetilde{\boldsymbol{a}}$ is uniquely determined by $x \in \mathcal{A}_{\phi}$ and we call it the canonical coefficient sequence of $x \in \mathcal{A}_{\phi}$. The next lemma (which basically follows from Lemma 2) characterizes the Fourier series of the canonical coefficient sequence of $x=\mathrm{T}_{\phi} \boldsymbol{a}$ in terms of the Fourier series of the sequence $\boldsymbol{a}$.

Lemma 3: Let $\boldsymbol{a} \in \ell^{2}$ and $x=\mathrm{T}_{\phi} \boldsymbol{a} \in \mathcal{A}_{\phi}$. Then the Fourier series of the canonical coefficient sequence $\widetilde{\boldsymbol{a}} \in \ell^{2}$ of $x$ is given by

$$
\widetilde{A}(\theta)=\sum_{n \in \mathbb{Z}} \widetilde{a}_{n} \mathrm{e}^{\mathrm{i} n \theta}=\left(\mathrm{P}_{\mathcal{M}_{\phi}} A\right)(\theta)=\left\{\begin{array}{ll}
A(\theta) & : \theta \in \mathcal{M}_{\phi} \\
0 & : \theta \in \mathcal{Z}_{\phi}
\end{array} .\right.
$$

Assume now that $x=\mathrm{T}_{\phi} \boldsymbol{a}$ is an arbitrary signal in $\mathcal{A}_{\phi}$ and assume that its generalized samples $\left\{c_{n}=\left\langle x, s_{n}\right\rangle\right\}_{n \in \mathbb{Z}}$ are given. Under which conditions on $s=\left\{s_{n}\right\}_{n \in \mathbb{Z}}$ will it be possible to reconstruct $x$ from $\left\{c_{n}\right\}_{n \in \mathbb{Z}}$ ? The answer is given by the following theorem.

Theorem 4: Let $\mathcal{A}_{\phi}$ be an atomic space of the form (1), spanned by a stationary frame sequence $\phi=\left\{\phi_{n}\right\}_{n \in \mathbb{Z}}$ with spectral density $\Phi_{\phi}$, and let $s=\left\{s_{n}\right\}_{n \in \mathbb{Z}}$ be a stationary sequence of sampling functions. Then every $x \in \mathcal{A}_{\phi}$ can be reconstructed from its samples $\left\{\left\langle x, s_{n}\right\rangle\right\}_{n \in \mathbb{Z}}$ by means of a bounded linear operator, if and only if

$$
\begin{array}{ll}
\text { 1) } & \mathcal{M}_{\phi} \subset \mathcal{M}_{s} \quad \text { and } \\
\text { 2) } & \Phi_{\phi, s}(\theta) \neq 0 \quad \text { for almost all } \theta \in \mathcal{M}_{\phi} .
\end{array}
$$

Remark: This theorem is a straight forward extension of the corresponding result under the assumption that $\phi$ is a Riesz basis for $\mathcal{A}_{\phi}$. Therefore, and because of the limited space, a proof is omitted. To recover $x$ from its samples, we note that because $x \in \mathcal{A}_{\phi}$ is completely determined by its canonical coefficients $\widetilde{\boldsymbol{a}}$, it is sufficient to determine $\widetilde{\boldsymbol{a}}$ from $\left\{c_{n}\right\}_{n \in \mathbb{Z}}$. This can be achieved by a linear filter $\widetilde{a}_{n}=\sum_{k \in \mathbb{Z}} \gamma_{k} c_{n-k}$ whose transfer function is given by

$$
\Gamma(\theta)=\sum_{k \in \mathbb{Z}} \gamma_{k} \mathrm{e}^{\mathrm{i} k \theta}=\left\{\begin{array}{ll}
1 / \Phi_{\phi, s}(\theta) & : \quad \theta \in \mathcal{M}_{\phi} \\
0 & : \quad \theta \in \mathcal{Z}_{\phi}
\end{array} .\right.
$$

Theorem 4 shows that if the sequence $s$ of sampling functions satisfies certain conditions, then every $x \in \mathcal{A}_{\phi}$ can be reconstructed

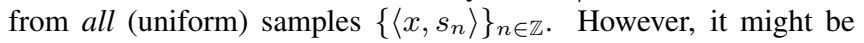
possible that actually less samples are necessary for signal recovery. This seems to be reasonable because to recover $x \in \mathcal{A}_{\phi}$, it is sufficient to determine its canonical coefficient sequence $\widetilde{\boldsymbol{a}}$. Intuitively, this should become easier as the support $\mathcal{M}_{\phi}$ of $\widetilde{A}$ becomes 
smaller. In particular, we are going to show that it is possible to recover $x \in \mathcal{A}_{\phi}$ from a subset of all generalized samples and that the "size" of this subset is determined by the size of $\mathcal{M}_{\phi}$. To this end, we define first the sparsity of an atomic space of the form (1).

Definition: Let $\phi=\left\{\phi_{n}\right\}_{n \in \mathbb{Z}}$ be a stationary frame sequence, let $\mathcal{A}_{\phi}=\overline{\operatorname{span}}\left\{\phi_{n}: n \in \mathbb{Z}\right\}$ be the corresponding atomic space, and let $s=\left\{s_{n}\right\}_{n \in \mathbb{Z}}$ be a sequence of sampling functions which satisfies the conditions of Theorem 4. If for any $x \in \mathcal{A}_{\phi}$ the sequence of generalized samples is denoted by $c=\left\{c_{n}=\left\langle x, s_{n}\right\rangle\right\}_{n \in \mathbb{Z}}$ then we say that $\mathcal{A}_{\phi}$ has an $(Q, N)$-sparsity pattern, if there exist $N$ subsequences of $c$ of the form

$$
\left\{c_{Q n+q}\right\}_{n \in \mathbb{Z}} \text { with } q \in J \subset\{0,1, \ldots, Q-1\} \text { and }|J|=N
$$

such that $x$ can be reconstructed from these signal samples only. Let $\mathcal{P}_{\mathcal{A}}$ denote the set off all $(Q, N)$ tuples such that $\mathcal{A}_{\phi}$ has a $(Q, N)$-sparsity pattern. Then the sparsity of $\mathcal{A}_{\phi}$ is defined as

$$
r\left(\mathcal{A}_{\phi}\right):=\sup \left\{\frac{Q-N}{Q}:(Q, N) \in \mathcal{P}_{\mathcal{A}}\right\} .
$$

By this definition, the sparsity of $\mathcal{A}_{\phi}$ is a positive number between zero and one. It is the ratio of redundant samples contained in all available samples. Thus $r\left(\mathcal{A}_{\phi}\right)=0$ if and only if $\phi$ is a Riesz basis for $\mathcal{A}_{\phi}$, i.e. if there are no redundant samples. On the other hand, values of $r\left(\mathcal{A}_{\phi}\right)$ close to 1 indicate a high ratio of redundant samples. For example, $r\left(\mathcal{A}_{\phi}\right)=0.9$ means that 9 out of 10 samples are redundant, or equivalently that only 1 out of 10 signal samples are necessary to recover every $x \in \mathcal{A}_{\phi}$.

We are going to show that the sparsity of $\mathcal{A}_{\phi}$ is determined by the spectral density of the generating sequence $\phi$.

Theorem 5: Let $\phi=\left\{\phi_{n}\right\}_{n \in \mathbb{Z}}$ be a stationary frame sequence in $\mathcal{H}$ with spectral density $\Phi_{\phi}$ and let $\mathcal{A}_{\phi}=\overline{\operatorname{span}}\left\{\phi_{n}: n \in \mathbb{Z}\right\}$. Then the sparsity of $\mathcal{A}_{\phi}$ is given by

$$
r\left(\mathcal{A}_{\phi}\right)=\frac{\lambda\left(\mathcal{Z}_{\phi}\right)}{2 \pi}=1-\frac{\lambda\left(\mathcal{M}_{\phi}\right)}{2 \pi} .
$$

Sketch of proof: Let $x \in \mathcal{A}_{\phi}$ be arbitrary. Then $x$ is uniquely determined by its canonical coefficient sequence, denoted by $\boldsymbol{a}$. To reconstruct $x$, we have to determine $\boldsymbol{a}$ from the samples $\boldsymbol{c}=\left\{c_{n}=\right.$ $\left.\left\langle x, s_{n}\right\rangle: n \in \mathbb{Z}\right\}$. Using (3), these samples can be written as

$$
\begin{aligned}
c_{n} & =\left\langle\sum_{k \in \mathbb{Z}} a_{k} \phi_{k}, s_{n}\right\rangle=\sum_{k \in \mathbb{Z}} a_{k} \frac{1}{2 \pi} \int_{0}^{2 \pi} \mathrm{e}^{\mathrm{i}(k-n) \theta} \Phi_{\phi, s}(\theta) \mathrm{d} \theta \\
& =\frac{1}{2 \pi} \int_{0}^{2 \pi} A(\theta) \Phi_{\phi, s}(\theta) \mathrm{e}^{-\mathrm{i} n \theta} \mathrm{d} \theta .
\end{aligned}
$$

Let $Q \in \mathbb{N}$ be arbitrary but fixed (the exact value of $Q$ is determined later). We define $Q$ subsequences of the sequences $\boldsymbol{a}$ and $\boldsymbol{c}$, respectively, as follows

$$
\begin{aligned}
\boldsymbol{c}^{(r)}: & =\left\{c_{n}^{(r)}:=c_{n Q+r}\right\}_{n \in \mathbb{Z}}, \quad r=0,1, \ldots, Q-1 \\
\boldsymbol{a}^{(q)}: & =\left\{a_{n}^{(q)}:=a_{n Q+q}\right\}_{n \in \mathbb{Z}}, \quad q=0,1, \ldots, Q-1 .
\end{aligned}
$$

The Fourier series of the subsequence $c^{(r)}$ is given by

$$
C^{(r)}(\theta)=\sum_{n \in \mathbb{Z}} c_{n Q+r} \mathrm{e}^{\mathrm{i} n \theta}=\left(\mathrm{D}_{Q} \mathrm{~S}^{r} C\right)(\theta)
$$

in terms of the Fourier series $C$ of $\boldsymbol{c}$, and a similar relation holds for the subsequences of $\boldsymbol{a}$. Finally, we stack the Fourier series of the subsequences (5) into vectors of length $Q$ as

$$
\boldsymbol{C}(\theta):=\left(\begin{array}{c}
C^{(0)}(\theta) \\
\vdots \\
C^{(Q-1)}(\theta)
\end{array}\right) \quad \text { and } \quad \boldsymbol{A}(\theta):=\left(\begin{array}{c}
A^{(0)}(\theta) \\
\vdots \\
A^{(Q-1)}(\theta)
\end{array}\right) .
$$

Now a straight forward calculation ${ }^{1}$, starting with (4), will show that

$$
\boldsymbol{C}(\theta)=\left(\mathrm{L}_{\boldsymbol{\Psi}} \boldsymbol{A}\right)(\theta)=\boldsymbol{\Psi}(\theta) \boldsymbol{A}(\theta), \quad \theta \in[0,2 \pi),
$$

where $\boldsymbol{\Psi}(\theta)$ is a self-adjoint matrix of size $Q \times Q$ whose entry in the $r$-th row and $q$-th column is given by

$$
[\boldsymbol{\Psi}(\theta)]_{r, q}=\left(\mathrm{D}_{Q} \mathrm{~S}^{r-q} \Phi_{\phi, s}\right)(\theta) .
$$

Using the definition of the decimation and shift operator $\mathrm{D}_{Q}$ and $\mathrm{S}$, respectively, the matrix $\boldsymbol{\Psi}$ and the vector $\boldsymbol{A}$ can be written as

$$
\begin{aligned}
& \boldsymbol{\Psi}(\theta)=\sum_{k=0}^{Q-1} \Phi_{\phi, s}\left(\frac{\theta}{Q}+k \frac{2 \pi}{Q}\right) \mathbf{v}_{k}(\theta) \mathbf{v}_{k}(\theta)^{*} \text { and } \\
& \boldsymbol{A}(\theta)=\sum_{k=0}^{Q-1} A\left(\frac{\theta}{Q}+k \frac{2 \pi}{Q}\right) \mathbf{v}_{k}(\theta)
\end{aligned}
$$

where $\mathbf{v}_{k}(\theta), k=0, \ldots, Q-1$ are length $Q$ vectors. Its entries in the $r$-th row are given by

$$
\left[\mathbf{v}_{k}(\theta)\right]_{r, 1}=\frac{1}{\sqrt{Q}} \exp \left[-\mathrm{i}(r-1)\left(\frac{\theta}{Q}+k \frac{2 \pi}{Q}\right)\right] .
$$

For every $\theta \in[0,2 \pi),\left\{\mathbf{v}_{k}(\theta)\right\}_{k=0}^{Q-1}$ is an orthonormal basis for $\mathbb{C}^{Q}$.

Now (7) shows that $\Psi(\theta)$ is the sum of $Q$ rank 1 matrices $\mathbf{v}_{k}(\theta) \mathbf{v}_{k}(\theta)^{*}$, and the coefficient of the $k$-th term in this sum is determined by the value of $\Phi_{\phi, s}$ in the interval $\left[k \frac{2 \pi}{Q},(k+1) \frac{2 \pi}{Q}\right)$. Since the sampling system $s$ satisfies the first condition of Theorem 4 and because $\mathcal{M}_{\phi, s}=\mathcal{M}_{\phi} \cap \mathcal{M}_{s}$, it follows that $\Phi_{\phi, s}(\theta)=0$ for all $\theta \in \mathcal{Z}_{\phi}$. Consequently, if $\lambda\left(\mathcal{Z}_{\phi}\right)>0$, then there exist indices $k \in\{0,1, \ldots, Q-1\}$ and $\theta \in[0,2 \pi)$ for which $\Phi_{\phi, s}([\theta+k 2 \pi] / Q)=0$. Then $\boldsymbol{\Psi}(\theta)$ has a rank smaller than $Q$ for some (or all) $\theta \in[0,2 \pi)$. Nevertheless, since $\boldsymbol{a}$ is assumed to be the canonical coefficient sequence of $x \in \mathcal{A}_{\phi}$, it follows from Lemma 3 that $A(\theta)=0$ for all $\theta$ where $\Phi_{\phi, s}(\theta)=0$. Consequently, (7) and (8) show that $\boldsymbol{A}(\theta) \in \mathscr{N}(\boldsymbol{\Psi}(\theta))^{\perp}$ for all $\theta \in \mathcal{T}$ such that the Fourier series $\boldsymbol{A}(\theta)$ of the canonical coefficient sequence can always be recovered from $\boldsymbol{C}(\theta)$ by $\boldsymbol{A}(\theta)=\boldsymbol{\Psi}(\theta)^{\dagger} \boldsymbol{C}(\theta)$ where $\boldsymbol{\Psi}(\theta)^{\dagger}$ denotes the Moore-Penrose pseudoinverse of $\boldsymbol{\Psi}(\theta)$.

In particular, if $Q$ is sufficiently large then there exist several intervals of the form $\left[k \frac{2 \pi}{Q},(k+1) \frac{2 \pi}{Q}\right)$ on which $\Phi_{\phi, s}(\theta)$ and $A(\theta)$ are identical to zero. Assume that this holds for $R$ out of all $Q$ intervals. Then the matrix $\Psi(\theta)$ will have a rank of at most $N:=Q-R$ for all $\theta \in[0,2 \pi)$, and its range is spanned by $N$ orthogonal vectors $\mathbf{v}_{k}(\theta)$. An upper bound on $R$ is apparently given by

$$
R=Q-N \leq \frac{\lambda\left(\mathcal{Z}_{\phi}\right)}{2 \pi / Q} .
$$

Since $\boldsymbol{\Psi}(\theta)$ has a rank of at most $N$, we may delete $R$ rows of $\boldsymbol{\Psi}(\theta)$ without reducing the rank of $\boldsymbol{\Psi}(\theta)$. This yields the matrix $\widetilde{\boldsymbol{\Psi}}(\theta)$ of size $N \times Q$ of the form

$$
\widetilde{\Psi}(\theta)=\sum_{k \in I} \Phi_{\phi, s}\left(\frac{\theta}{Q}+k \frac{2 \pi}{Q}\right) \widetilde{\mathbf{v}}_{k}(\theta) \mathbf{v}_{k}(\theta)^{*}
$$

where $I \subset\{0,1,2, \ldots, Q-1\}$ with $|I|=N$, and $\widetilde{\mathbf{v}}_{k}(\theta)$ is a length $N$ vector which is obtained by deleting $R$ rows of $\mathbf{v}_{k}(\theta)$.

\footnotetext{
${ }^{1}$ which may be found in the proof of Theorem 3 of [3]
} 
Of course, in general it will depend on the set $I$ which rows of the vectors $\left\{\mathbf{v}_{k}\right\}_{k \in I}$ can be deleted such that $\widetilde{\Psi}(\theta)$ still has rank $N$. However, there always exists at least one set of $R$ columns which can be deleted such that $\widetilde{\Psi}$ still has rank $N$. Equation (6) shows that the deletion of rows of $\boldsymbol{\Psi}(\theta)$ corresponds to leaving out the corresponding substreams of signal samples. Thus, overall we have

$$
\widetilde{\boldsymbol{C}}(\theta)=\widetilde{\boldsymbol{\Psi}}(\theta) \boldsymbol{A}(\theta), \quad \theta \in[0,2 \pi),
$$

wherein $\widetilde{\boldsymbol{C}}(\theta)$ contains the Fourier series of $N$ out of $Q$ substreams $\boldsymbol{c}^{(r)}$ of (4). Moreover, (11) shows that still $\boldsymbol{A}(\theta) \in \mathscr{N}(\widetilde{\boldsymbol{\Psi}}(\theta))^{\perp}$ such that it is possible to reconstruct $\boldsymbol{A}(\theta)$ from $\widetilde{\boldsymbol{C}}(\theta)$ by

$$
\boldsymbol{A}(\theta)=\widetilde{\boldsymbol{\Psi}}(\theta)^{\dagger} \widetilde{\boldsymbol{C}}(\theta)
$$

From $\boldsymbol{A}(\theta)$ the canonical coefficient sequence $\boldsymbol{a}$ of $x \in \mathcal{A}_{\phi}$ can be determined and thus $x$ can be reconstructed from only $N$ out of $Q$ substreams of samples. In other words, we have shown that $\mathcal{A}_{\phi}$ has a $(Q, N)$ sparsity pattern, and it follows from (10) that the sparsity of $\mathcal{A}_{\phi}$ is upper bounded by $r\left(\mathcal{A}_{\phi}\right) \leq \lambda\left(\mathcal{Z}_{\phi}\right) / 2 \pi$. To finish the proof, it remains to show that the number $R$, as defined above, becomes as close as desired to value on the right hand side of (10), by choosing $Q$ sufficiently large. However, this seems to be straight forward by the above construction, such that the details are omitted here.

\section{SIGNAL RECOVERY}

The proof of Theorem 5 shows how a sparse signal $x \in \mathcal{A}_{\phi}$ can be reconstructed from only $N \geq\left[1-r\left(\mathcal{A}_{\phi}\right)\right] Q$ out of $Q$ substreams of the uniform signal samples $c_{n}=\left\langle x, s_{n}\right\rangle, n \in \mathbb{Z}$. In the Fourier domain, signal recovery is given by (12), and we need to determine the pseudoinverse $\widetilde{\boldsymbol{\Psi}}(\theta)^{\dagger}$ of (11). The matrix $\widetilde{\boldsymbol{\Psi}}(\theta)$ was obtained from $\Psi(\theta)$ by deleting $R$ rows in such a way that both matrices have the same maximum rank $N$. In view of (11) this is equivalent to require that the set $\left\{\widetilde{\mathbf{v}}_{k}(\theta)\right\}_{k \in I}$ forms a Riesz basis for $\mathbb{C}^{N}$ for all $\theta \in \mathcal{T}$. Let the set $\left\{\widetilde{\mathbf{w}}_{k}(\theta)\right\}_{k \in I}$ of vectors in $\mathbb{C}^{N}$ be the corresponding dual Riesz basis. Then it is easy to see that

$$
\widetilde{\mathbf{\Psi}}(\theta)^{\dagger}=\sum_{k \in I} \Phi_{\phi, s}\left(\frac{\theta}{Q}+k \frac{2 \pi}{Q}\right)^{\dagger} \mathbf{v}_{k}(\theta) \widetilde{\mathbf{w}}_{k}(\theta)^{*},
$$

where $\Phi(\theta)^{\dagger}=1 / \Phi(\theta)$ for all $\theta$ where $\Phi(\theta) \neq 0$ and $\Phi(\theta)^{\dagger}=0$ for all $\theta$ where $\Phi(\theta)=0$. To determine $\left\{\widetilde{\mathbf{w}}_{k}(\theta)\right\}_{k \in I}$, notice that the vectors $\mathbf{v}_{k}(\theta)$ given in (9) can be written as $\mathbf{v}_{k}(\theta)=\mathbf{D}(\theta) \mathbf{f}_{k}$, where $\mathbf{D}(\theta)$ is a unitary $Q \times Q$ diagonal matrix with entries $[\mathbf{D}(\theta)]_{r, r}=$ $\exp (-\mathrm{i}[r-1] \theta / Q)$, and $\mathbf{f}_{k}$ is the $(k+1)$ th column of the usual DFT matrix of size $Q \times Q$, i.e.

$$
\left[\mathbf{f}_{k}\right]_{r, 1}=\frac{1}{\sqrt{Q}} \exp \left(-\mathrm{i} \frac{2 \pi}{Q} k[r-1]\right), \quad r=1,2, \ldots, Q .
$$

Now we collect the vectors $\left\{\mathbf{v}_{k}(\theta)\right\}_{k \in I}$ in a $Q \times N$ matrix $\mathbf{V}_{I}(\theta):=$ $\left[\mathbf{v}_{k_{1}}(\theta), \ldots, \mathbf{v}_{k_{N}}(\theta)\right]=\mathbf{D}(\theta) \mathbf{F}_{I}$, where $\mathbf{F}_{I}$ is the $Q \times N$ matrix with columns $\left\{\mathbf{f}_{k}\right\}_{k \in I}$. Moreover, if one deletes $R=Q-N$ columns of $\mathbf{V}_{I}$, one obtains the $N \times N$ matrix $\widetilde{\mathbf{V}}_{I}(\theta)=\widetilde{\mathbf{D}}(\theta) \widetilde{\mathbf{F}}_{I}$ whose columns are $\left\{\widetilde{\mathbf{v}}_{k}(\theta)\right\}_{k \in I}$. Moreover, $\widetilde{\mathbf{D}}(\theta)$ is an $N \times N$ unitary diagonal matrix, and $\widetilde{\mathbf{F}}_{I}$ is a constant $N \times N$ matrix obtained from $\mathbf{F}_{I}$ by deleting $R$ rows. Therewith, the vectors $\left\{\widetilde{\mathbf{w}}_{k}(\theta)^{*}\right\}_{k \in I}$ of the dual Riesz basis of $\left\{\widetilde{\mathbf{v}}_{k}(\theta)\right\}_{k \in I}$ are obtained as the rows of the inverse of $\widetilde{\mathbf{V}}_{I}(\theta)$ which is given by

$$
\widetilde{\mathbf{W}}_{I}(\theta):=\widetilde{\mathbf{V}}_{I}(\theta)^{-1}=\widetilde{\mathbf{F}}_{I}^{-1} \widetilde{\mathbf{D}}(\theta)^{*} .
$$

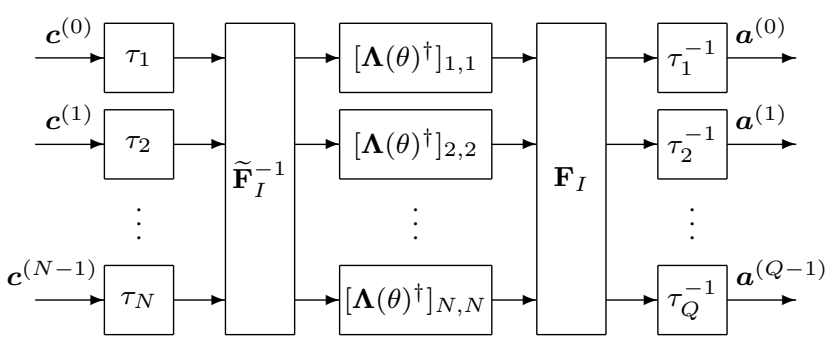

Fig. 1. Reconstruction of all $Q$ substreams of signal coefficients from $N \leq Q$ substreams of generalized signal samples.

Using the so obtained $\widetilde{\mathbf{w}}_{k}(\theta)^{*}$ in (13), one obtains finally

$$
\widetilde{\mathbf{\Psi}}(\theta)^{\dagger}=\mathbf{D}(\theta) \mathbf{F}_{I} \mathbf{\Lambda}(\theta)^{\dagger} \widetilde{\mathbf{F}}_{I}^{-1} \widetilde{\mathbf{D}}(\theta)^{*}
$$

where $\boldsymbol{\Lambda}(\theta)^{\dagger}$ is an $N \times N$ diagonal matrix whose entries are given by the coefficients $\Phi_{\phi, s}([\theta+k 2 \pi] / Q)^{\dagger}$ with $k \in I$ in (13).

Overall, signal recovery in the time domain may be illustrated as in Fig. 1. On the left, we have $N$ substreams of signal samples as an input. The blocks with $\tau$ 's symbolize multiplications with the corresponding exponential functions on the diagonal of the matrices $\widetilde{\mathbf{D}}(\theta)^{*}$ and $\mathbf{D}(\theta)$ in the Fourier domain. This corresponds to delaying the signal samples in the time domain. The two large blocks stand for symbol-wise multiplications with the matrices $\widetilde{\mathbf{F}}_{I}^{-1}$ and $\mathbf{F}_{I}$, respectively, whereas the blocks in the middle are linear filters whose transfer functions are given by the entries of the diagonal ma$\operatorname{trix} \boldsymbol{\Lambda}(\theta)^{\dagger}$.

\section{RELATION TO PRIOR WORK AND EXTENSIONS}

The problem of signal recovery from sparse samples where considered in a vast number of publications for a variety of different settings, e.g.: (a) finite dimensional spaces [12], (b) multiband signals $[13,14,15],(c)$ general shift-invariant space [16, 17], $(d)$ unions of subspaces [18], or $(e)$ general Hilbert spaces [19]. The present paper generalizes in particular results concerning sampling and reconstruction of multiband signals and of signals in shift-invariant spaces. The setting considered in these papers is obtained by assuming that the unitary operator $U$, which generates the spanning sequence $\phi$ of the signal space $\mathcal{A}_{\phi}$, is the translation operator. Apart from the fact that our setting is much more general, it provides also an alternative and different derivation for the multiband setting. Sampling in shiftinvariant spaces with frame generators was considered in [20], but without characterizing the amount of sparsity in these spaces.

The present paper may also be considered as a contribution to the recent interest in defining an appropriate notion of redundancy for frames, see, e.g., [21, 22, 23]. Although the notion of redundancy in these papers, means something slightly different than sparsity, as in the present paper, the sparsity $r\left(\mathcal{A}_{\phi}\right)$, as defined here, also gives of quantitative notion of the overcompletness of the sequence $\phi$.

In this paper, it was assumed that the support $\mathcal{M}_{\phi}$ of the spectral density $\Phi_{\phi}$ of the spanning sequence $\left\{\phi_{n}\right\}_{n \in \mathbb{Z}}$ is known. Only with this knowledge, we are able to determine the necessary subsequences of signal samples, to reconstruct every $x \in \mathcal{A}$. However, if $\mathcal{M}_{\phi}$ is not known but only its size $\lambda\left(\mathcal{M}_{\phi}\right)$, then one may apply methods from compressed sensing [15], to first recover the support of $\Phi_{\phi}$ and then to recover $x \in \mathcal{A}$ from the signal samples. 


\section{REFERENCES}

[1] C. de Boor, R. DeVore, and A. Ron, "The structure of finitely generated shift-invariant spaces in $L^{2}\left(\mathbb{R}^{d}\right)$," J. Funct. Anal., vol. 119, no. 1, pp. 37-78, 1994.

[2] A. Aldroubi, "Oblique projections in atomic spaces," Proc. Amer. Math. Soc., vol. 124, no. 7, pp. 2051-2060, Jul. 1996.

[3] V. Pohl and H. Boche, "U-Invariant Sampling and Reconstruction in Atomic Spaces with Multiple Generators," IEEE Trans. Signal Process., vol. 60, no. 7, pp. 3506-3519, Jul. 2012.

[4] H. Boche and U. J. Mönich, "Sampling of Deterministic Signals and Systems," IEEE Trans. Signal Process., vol. 59, no. 6, pp. 2101-2111, May 2011.

[5] — , "Unboundedness of Thresholding and Quantization for Bandlimited Signals," Signal Processing, vol. 92, no. 12, pp. 2821-2829, Dec. 2012.

[6] Y. A. Rozanov, Stationary Random Processes. San Francisco: Holden-Day, 1967.

[7] O. Christensen, An Introduction to Frames and Riesz Bases. Bosten: Birkhäuser, 2003.

[8] T. Michaeli, V. Pohl, and Y. C. Eldar, "U-Invariant Sampling: Extrapolation and Causal Interpolation from Generalized Samples," IEEE Trans. Signal Process., vol. 59, no. 5, pp. 20852100, May 2011.

[9] J. Benedetto and S. Li, "The Theory of Multiresolution Analysis Frames and Applications to Filter Banks," Appl. Comp. Harm. Anal., vol. 5, no. 4, pp. 389-427, 1998.

[10] M. Unser, "Sampling - 50 Years After Shannon," Proc. IEEE, vol. 88, no. 4, pp. 569-587, Apr. 2000.

[11] V. Pohl, "On Causal Estimation from Bandlimited Stochastic Sequences," IEEE Trans. Inf. Theory, vol. 57, no. 9, pp. 54365443, Aug. 2011.

[12] D. L. Donoho and X. Huo, "Uncertainty Principle and Ideal Atomic Decomposition," IEEE Trans. Inf. Theory, vol. 47, no. 7, pp. 2845-2862, 2001.

[13] L. Bezuglaya and V. Katsnelson, "The Sampling Theorem for Functions with Limited Multi-Band Spectrum I," Z. Anal. Anwendungen, vol. 12, pp. 511-534, 1993.
[14] R. Venkataramani and Y. Bresler, "Perfect Reconstruction Formulas and Bounds on Aliasing Error in Sub-Nyquist Nonuniform Sampling of Multiband Signals," IEEE Trans. Inf. Theory, vol. 46, no. 6, pp. 2173-2183, Sep. 2000.

[15] M. Mishali and Y. C. Eldar, "Blind Multiband Signal Reconstruction: Compressed Sensing for Analog Signals," IEEE Trans. Signal Process., vol. 57, no. 3, pp. 993-1009, Mar. 2009.

[16] Y. C. Eldar, "Compressed Sensing of Analog Signals in ShiftInvariant Spaces," IEEE Trans. Signal Process., vol. 57, no. 8, pp. 2986-2997, Aug. 2009.

[17] A. G. García, M. A. Hernández-Medina, and G. Pérez-Villalón, "Oversampling in Shift-Invariant Spaces With a Rational Sampling Period," IEEE Trans. Signal Process., vol. 57, no. 9, pp. 3442-3449, Sep. 2009.

[18] Y. M. Lu and M. N. Do, "A Theory for Sampling Signals From a Union of Subspaces," IEEE Trans. Signal Process., vol. 56, no. 6, pp. 2334-2345, Jun. 2008.

[19] G. Pope and H. Bölcskei, "Sparse Signal Recovery in Hilbert Spaces," in Proc. IEEE Intern. Symp. on Information Theory (ISIT), Boston, MA, USA, Jul. 2012, pp. 1463-1467.

[20] A. G. García, J. M. Kim, K. H. Kwon, and G. J. Yoon, "MultiChannel Sampling on Shift-Invariant Spaces with Frame Generators," Int. J. Wavelets Multiresolut. Inf. Process, vol. 10, no. 1, pp. 41-60, Jan. 2012.

[21] R. Balan, P. G. Casazza, C. Heil, and Z. Landau, "Deficits and excesses of frames," Adv. Comput. Math., vol. 18, no. 2-4, pp. 93-116, 2003.

[22] B. G. Bodmann, P. G. Casazza, and G. Kutyniok, "A Quantitative Notion of Redundancy for Finite Frames," Appl. Comput. Harmon. Anal., vol. 30, no. 3, pp. 348-362, May 2011.

[23] J. Cahill, P. G. Casazza, and A. Heinecke, "A Notion of Redundancy for Infinite Frames," in Proc. 9th Intern. Conf. on Sampling Theory and Applications (SAMPTA), Singapore, May 2011. 